\title{
Simulation of a discharge electrode needle for particle charging in an electrostatic precipitator
}

\author{
Sebastian C.-Beckers ${ }^{1 *}$, Julian Pawlik ${ }^{2}$, Hikmet Eren ${ }^{1}$, Adam Sanaf $^{1}$, Jürgen Kiel ${ }^{1}$ \\ ${ }^{1}$ FMDauto-Institut, Düsseldorf University of Applied Sciences, Münsterstr. 156, 40476 Düsseldorf, Germany; \\ *sebastian.beckers@hs-duesseldorf.de \\ ${ }^{2}$ getAir GmbH, Krefelder Str. 670, 41066 Mönchengladbach, Germany
}

\begin{abstract}
This paper describes a model approach for the simulation of a discharge electrode (DE) needle to charge particles using positive ions in an electrostatic precipitator. This includes the simulation of the electrostatic field, the space charge field of the ions and the flow field at the DE needle. The interactions of the fields, e.g. the reaction of the space charge on the electrostatic field or the electric wind are also considered in the model. To simplify and accelerate the simulation, a radial symmetry around the DE needle is partly assumed. The results of the simulation are validated by comparing the experimentally determined current-voltage characteristic with the simulated one, which show a satisfying correlation. Therefore, this model can be used as a basis for future particle flight simulation and further investigations.
\end{abstract}

\section{Introduction}

In residential applications, two-stage electrostatic precipitators (ESPs) are mainly used to separate harmful particles from the air. Particles entering the filter are first charged in the ioniser by an ion field based on a corona discharge and then separated in a subsequent filter stage by an electrostatic field (Coulomb's law) on the electrodes of the collector.

Although this filtering process is very efficient, it has the major disadvantage that it generates ozone during operation [1] [2]. Ozone can be harmful to human health when inhaled, therefore the WHO (Air Quality Guidelines Global Update 2005) sets a limit value of $50 \mathrm{ppb}$ (parts per billion) for an average exposure of eight hours.

A very effective method to minimize the ozone concentration is to reduce the corona plasma region at the discharge electrode (DE) within the ioniser [3] [4], where the ozone production process takes place. Consequently, the development of DEs is geared towards ever smaller dimensions [5]. The shape and arrangement of these DEs can be very different for each application, which makes a generally valid analytical mathematical description difficult and therefore requires numerical modelling.

Experimental studies on particle separation and ozone generation have shown good results with particle charging by a DE needle [6]. Therefore, the modelling of this approach is described in the following.

\section{Experimental setup}

The experimental setup used in this study consists of a stainless-steel DE needle with a radius of curvature of $55 \mu \mathrm{m}$ at the tip and a round grid arranged at a distance of $50 \mathrm{~mm}$ as a ground electrode with a diameter of $85 \mathrm{~mm}$, as shown in Fig. (1). The DE needle is centered by a holder on the rotation axis and protrudes $4 \mathrm{~mm}$ from it.

Furthermore, the DE needle is raised to a positive voltage potential by a high-voltage source of the company FUG (HCP35-20000) and the grid is connected to an electrical grounding. By using this configuration, it is possible to set and measure voltages as well as currents. Thus, the voltage-current characteric of the DE needle can be analysed.

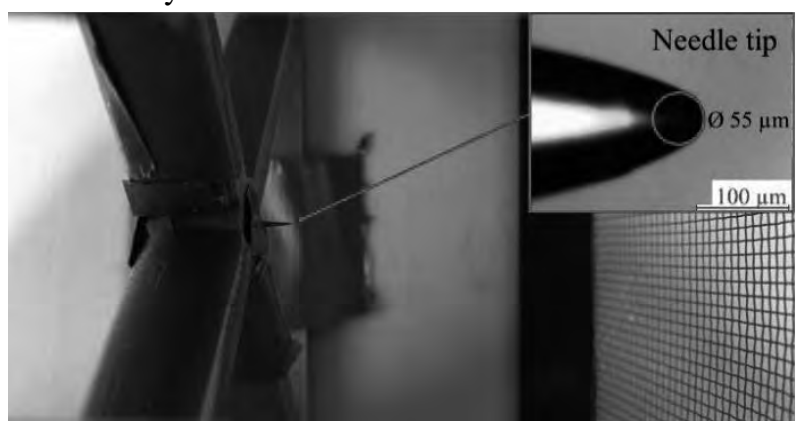

Figure 1: Test setup with the stainless-steel discharge electrode needle in the middle.

\section{Model}

\subsection{Model approach}

In order to implement the simulation of a DE needle, not only the electrostatic field, but also the flow and space charge field must be modelled. 
Furthermore, the interactions of the different fields are considered in the model. For example, the reaction of the space charge density to the electrostatic field as well as the electric wind as an impact of the electrohydrodynamic (EHD) effect are taken into consideration. Fig. (2) gives an overview of the model approach.

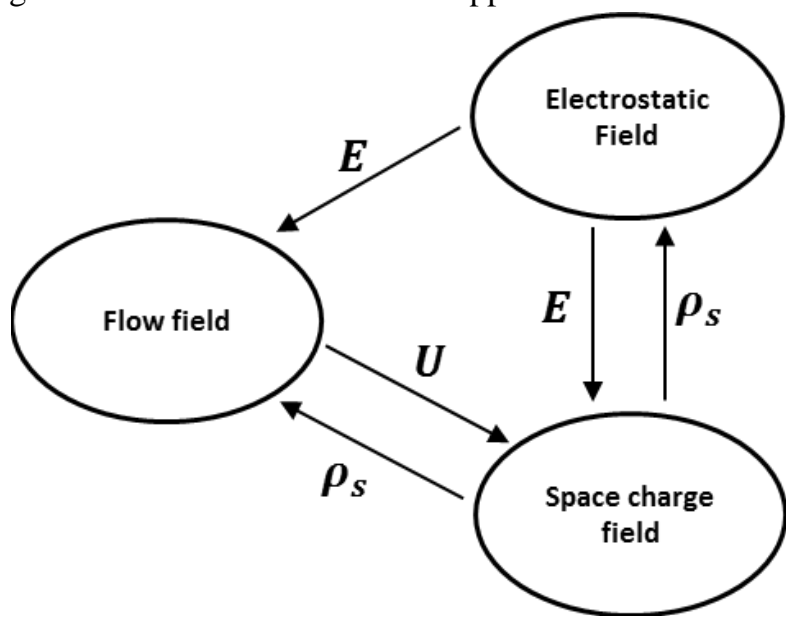

Figure 2: Overview of the simulated fields and their interactions.

To simplify the simulation, the geometry of the DE needle is modelled as a simple composition of a truncated cone with an outer diameter of $0.6 \mathrm{~mm}$ and a length of $4 \mathrm{~mm}$ and a semi-sphere with a diameter of $55 \mu \mathrm{m}$ as needle tip, see Fig. (3).

The holder of the DE needle is also simplified as a cylinder with a diameter of $11.4 \mathrm{~mm}$ and a length of $10 \mathrm{~mm}$, as is the measuring chamber with a diameter of $85 \mathrm{~mm}$ and a length of $64 \mathrm{~mm}$. The geometry of the grid at the exit of the measuring chamber is neglected.

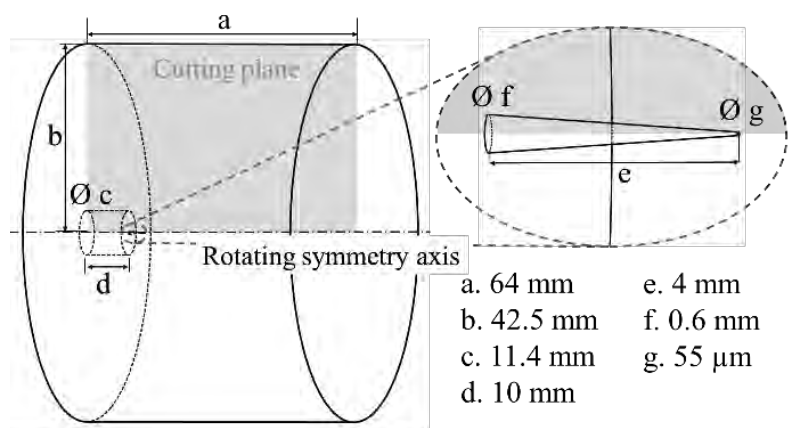

Figure 3: Simplified geometric model of the experiment.

In addition, a radial symmetry around the DE needle is assumed for the simulation of the space charge field and the flow field respectively, as shown in Fig. (4).

The micromechanisms of the corona plasma region are not simulated but the resulting convection current of the space charges are. As a further simplification of the procedure, the corona plasma region is placed on the DE needle tip surface. A positive corona and thus a positive convection current (positive ions) are assumed.

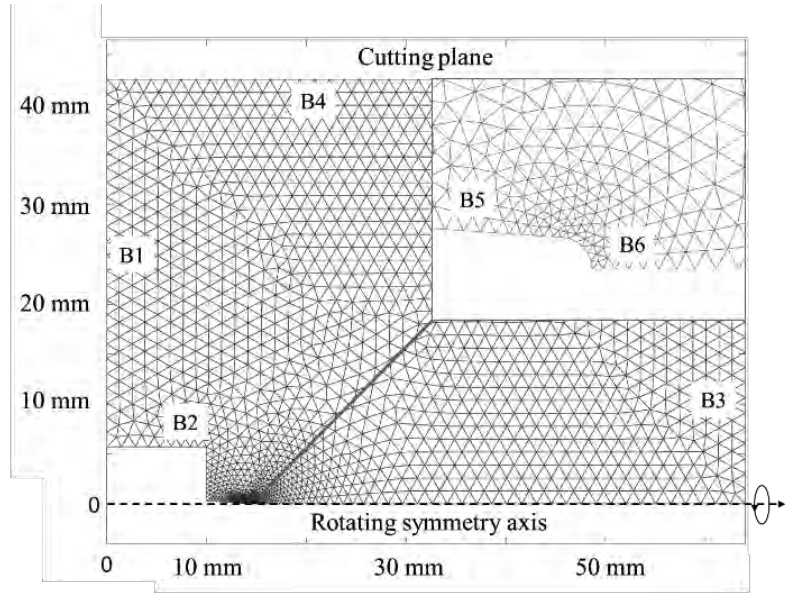

Figure 4: Geometry and meshing of the cutting plane with the boundary numbers.

\subsection{Electrostatic field}

The electrostatic field can be described mathematically using the following equation of Poisson [7]:

$$
\Delta \mathrm{V}=-\frac{\rho_{\mathrm{s}}}{\varepsilon_{0} \varepsilon_{r}}
$$

where $\mathrm{V}$ is the voltage potential $(V), \rho_{\mathrm{s}}$ the space charge density $\left(A s \mathrm{~m}^{-3}\right), \varepsilon_{0}$ the permittivity of the vacuum (8.85 $\cdot 10^{-12} A s V^{-1} m^{-1}$ ) and $\varepsilon_{r}$ the relative permittivity (approx. 1 for air).

Due to the very fine DE electrode tip, which is strongly curved in contrast to the grid ground electrode, a very inhomogeneous electrostatic field is created. In order to cope with this and take all effects into account, it is simulated in three dimensions. For the simulation itself, as well as the meshing, the Partial Differential Equation Toolbox (PDE-Tool) in MATLAB ${ }^{\circledR}$ is used. The total amount of tetrahedral cells used in the simulation mesh is 48811 .

The tool's integrated solver calculates the solution using an FEM algorithm, assuming the following boundary conditions, where the locations can be obtained from Fig. (4).

\begin{tabular}{lll}
\hline Boundary & Description & Value \\
\hline B5, B6 & Potential at DE needle & $V_{S E}=V_{0}+\Delta V$ \\
\hline B3 & Potential at grid & $V_{\text {grid }}=0$ (ground) \\
\hline \multicolumn{2}{c}{ Table 1: } & Boundary conditions of the electrostatic field.
\end{tabular}

The voltage potential at the DE needle $\mathrm{V}_{S E}$ is composed 
of the breakdown voltage $V_{0}$ which corresponds to the initial voltage of the corona discharge, and a correction value $\Delta \mathrm{V}$ which is described in detail in Chpt. (2.5).

\subsection{Space charge field}

The simulation of the space charge field is based on the formula of White [8], which describes the current density $\boldsymbol{J}\left(A \mathrm{~m}^{-2}\right)$ considering the convection and diffusion charge transport components.

$$
\begin{gathered}
\nabla \cdot \boldsymbol{J}=0 \\
\boldsymbol{J}=\left(b_{i} \boldsymbol{E}+\boldsymbol{U}\right) \rho_{s}-D_{i} \nabla \rho_{s}
\end{gathered}
$$

The convection part of Eq. (3) shows the coupling to the electrostatic field $\boldsymbol{E}\left(V \mathrm{~m}^{-1}\right)$ and to the velocity field $\boldsymbol{U}$ $\left(m s^{-1}\right)$. The quantities $\rho_{s}$ and $b_{i}$ represent the space charge density $\left(A s \mathrm{~m}^{-3}\right)$ and the ion mobility $\left(m V^{-1} s^{-1}\right)$ respectively. The latter is assumed as a constant with the value $b_{i}=1.85 \cdot 10^{-4} \mathrm{mV}^{-1} \mathrm{~s}^{-1}$ [9].

The diffusion part of Eq. (3) consists of the local gradient of the space charge $\nabla \rho_{s}\left(A s \mathrm{~m}^{-4}\right)$, and the ionic diffusion coefficient $D_{i}\left(m^{2} s^{-1}\right)$, which can be estimated using the following formula [10]:

$$
D_{i}=\left(b_{i} k T\right) / e
$$

where $k$ is the Boltzmann's constant $\left(1.38 \cdot 10^{-23} J^{-1}\right), \quad e$ the elementary charge (1.6 $\cdot 10^{-19} A s$ ) and $T$ the temperature $(K)$.

Since the geometry can be assumed to be approximately rotationally symmetrical, the simulation area for modelling the space charge density can be reduced to a two-dimensional cutting plane, see Fig. (3) and (4). As with the electrostatic field, the automatic mesher of the PDE-Tool is used for the grid generation of the two-dimensional solution area. The two-dimensional grid used has 4512 triangular cells.

The solution of Eq. (2) which describes the space charge transport is achieved by using the Finite Volume Method (FVM) in MATLAB ${ }^{\circledR}$. In order to accomplish that, the solution area $(\Omega)$ is divided into many subareas $\left(\Omega_{i}\right)$ (finite volumes) and the current density at the interfaces is balanced:

$$
\int_{\Omega_{i}} \nabla \cdot\left(\left(b_{i} \boldsymbol{E}+\boldsymbol{U}\right) \rho_{s}-D_{i} \nabla \rho_{s}\right) d \Omega_{i}=0
$$

Due to the Gaussian integral theorem and the assumption that the values on the cell-face are uniform over the entire face, Eq. (5) can be brought into a discrete form [11]:

$$
\sum_{f}\left[\left[\left(b_{i} \boldsymbol{E}+\boldsymbol{U}\right) \cdot \boldsymbol{n}\right]_{f} \rho_{s f}-\left(D_{i} \frac{\partial \rho_{s}}{\partial n}\right)_{f}\right] A_{f}=0
$$

where the index $f$ represents the face, $\boldsymbol{n}$ the normal vector and $A_{f}$ the area of the face.

The convection term of Eq. (6) is calculated according to Long [12] using the second order Upwind Difference Method ( $2^{\text {nd }}$ UDM). In this method, a Taylor series approach is used to project the respective space charge density onto the center of the intersection face $(F)$, see also Fig. (5).

$$
\sum_{f}\left[\left(b_{i} \boldsymbol{E}+\boldsymbol{U}\right) \cdot \boldsymbol{n}\right]_{f} \rho_{s F, f} A_{f}
$$

The projected space charge density $\rho_{S F, f}$ can be calculated using the $2^{\text {nd }}$ UDM with the following case distinction:

$$
\begin{aligned}
& \boldsymbol{\rho}_{\boldsymbol{s} \boldsymbol{F}, \boldsymbol{f}} \\
& =\left\{\begin{array}{l}
\rho_{s_{C}}+\nabla \rho_{s_{C}} \cdot \boldsymbol{d}_{C F} \text { if }\left(\left(b_{i} \boldsymbol{E}+\boldsymbol{U}\right) \cdot \boldsymbol{n}\right)_{F, f}>0 \\
\rho_{s_{N}}+\nabla \rho_{s_{N}} \cdot \boldsymbol{d}_{N F} \text { if }\left(\left(b_{i} \boldsymbol{E}+\boldsymbol{U}\right) \cdot \boldsymbol{n}\right)_{F, f}<0
\end{array}\right\}
\end{aligned}
$$

where $\nabla \rho_{s_{C}}$ is the local gradient of space charge densities of the cell and $\nabla \rho_{s_{N}}$ is the one of the neighbouring cell. In this equation, the vectors $\boldsymbol{d}_{C F}$ and $\boldsymbol{d}_{N F}$ represent the distance vectors between the centers of the particular cell ( $C$ and $N$ ) and the center point of the intersection face $(F)$.

The diffusion term in Eq. (6) is implicitly calculated in this study using the space charge field.

$$
\sum_{f}-\left(D_{i} \frac{\partial \rho_{s}}{\partial n}\right)_{f} A_{f}
$$

Following the approach of Long [12], the gradient of space charge density in the diffusion term is determined by projected substitute points for the space charge density of the cell $\rho_{s C^{\prime}}$ and the neighboring cell $\rho_{S N^{\prime}}$ as well as the projected substitute point on the intersection face $\rho_{S F^{\prime}}$. These three substitute points are determined by the following equations:

$$
\begin{aligned}
& \rho_{S C^{\prime}}=\rho_{S C}+\nabla \rho_{S C} \cdot \boldsymbol{d}_{C C^{\prime}} \\
& \rho_{S N^{\prime}}=\rho_{S N}+\nabla \rho_{S N} \cdot \boldsymbol{d}_{N N^{\prime}}
\end{aligned}
$$

and

$$
\begin{gathered}
\rho_{S F C}=\rho_{S C}+\nabla \rho_{S C} \cdot \boldsymbol{d}_{C F} \\
\rho_{S F N}=\rho_{S N}+\nabla \rho_{S N} \cdot \boldsymbol{d}_{N F}
\end{gathered}
$$




$$
\rho_{S F}=\frac{\rho_{S F C}+\rho_{S F N}}{2}
$$

where $\nabla \rho_{s}$ is the local gradient of the respective cell and $\boldsymbol{d}$ is the respective difference vector between the corresponding points in the indices. $\rho_{S F C}$ and $\rho_{S F N}$ represent space charge density values projected from the centers of the cell $(\mathrm{C})$ and the neighbouring cell $(\mathrm{N})$ to the center point of the intersection face $(F)$.

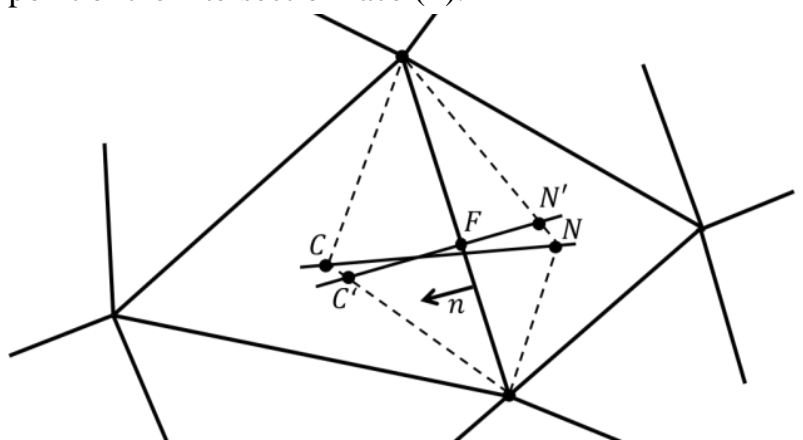

Figure 5: Demonstration of the gradient calculation between adjacent mesh cells.

Based on these three substitute points of the space charge density (Eq. (12) to Eq. (14)), the mean slope can be determined by linear interpolation. The mean slope then corresponds to the gradient at the intersection face of both cells.

The convection and diffusion term of space charge transport shown in Eq. (7) and Eq. (9) can then be expressed in a simple form:

$$
\sum_{f} a_{\text {conv }_{f}} \rho_{s, f}=\sum_{f}\left(b_{\text {conv }_{f}}-b_{d i f f_{f}}\right)
$$

where

$$
\begin{gathered}
a_{\text {conv }_{f}}=\left[\left(b_{i} \boldsymbol{E}+\boldsymbol{U}\right) \cdot \boldsymbol{n}\right]_{f} A_{f} \\
b_{\text {conv }_{f}}=-a_{\text {conv }_{f}}\left(\nabla \rho_{s_{i}} \cdot \boldsymbol{d}_{i j}\right)_{f} \\
b_{\text {diff } f_{f}}=-\left(D_{i} \frac{\partial \rho_{s}}{\partial n}\right)_{f} A_{f}
\end{gathered}
$$

The quantities $\nabla \rho_{s_{i}}$ and $\boldsymbol{d}_{i j}$ refer to the case distinction of the $2^{\text {nd }} U D M$ in Eq. (8).

If Eq. (15) is applied to all cells in the solution area it yields a linear system of equations in the form:

$$
\boldsymbol{A} \cdot \boldsymbol{\rho}_{\mathrm{s}}=\boldsymbol{B}
$$

which is then solved using the method of least squares (lqslin function) in MATLAB ${ }^{\circ}$.

The boundary conditions used for the simulation of the space charge field are given below.

\begin{tabular}{lll}
\hline Boundary & Description & Value \\
\hline B6 & $\begin{array}{l}\text { Current density } \\
\text { input }\end{array}$ & $\boldsymbol{J} \cdot \boldsymbol{n}=\frac{I_{0}}{A_{\text {out }}}$ \\
\hline B1, B3 & $\begin{array}{l}\text { Current density } \\
\text { output }\end{array}$ & $\boldsymbol{J} \cdot \boldsymbol{n}=\left[\left(b_{i} \boldsymbol{E}+\boldsymbol{U}\right) \cdot \boldsymbol{n}\right] \rho_{s}$ \\
\hline B2, B4, B5 & Wall & $\boldsymbol{J} \cdot \boldsymbol{n}=0$
\end{tabular}

Table 2: Boundary conditions of the space charge field.

The current value $I_{0}$ in the boundary condition of the input current density represents an input parameter of the model and must be distributed over the entire outlet surface $A_{\text {out }}$ of the DE needle tip.

For the boundary condition of the output current density, only the convection component is taken into account, due to the assumption that the change of space charge density near the surface of the output is neglectable.

\subsection{Flow field}

The flow field in an electrostatic precipitator which can be modelled according to [13 - 15] by the time-averaged Navier-Stokes equation for incompressible fluids with the standard $k$ - $\epsilon$ turbulence model [16]:

$$
\nabla \cdot \boldsymbol{U}=0
$$

$$
\rho_{F}(\boldsymbol{U} \cdot \nabla) \boldsymbol{U}-\left(\mu+\mu_{T}\right) \Delta \boldsymbol{U}=-\nabla \mathrm{p}+\rho_{F} \mathbf{g}+\boldsymbol{F}_{\boldsymbol{E} \boldsymbol{H} \boldsymbol{D}}
$$

where $\rho_{F}$ is the fluid densitiy $\left(\mathrm{kg} \mathrm{m}^{-3}\right), \mu$ the laminar viscosity $\left(\mathrm{kg} \mathrm{m}^{-1} \mathrm{~s}^{-1}\right), \mu_{T}$ the turbulent viscosity of the $\kappa-\epsilon$ turbulence model $\left(\mathrm{kg} \mathrm{m}^{-1} \mathrm{~s}^{-1}\right)$, p the fluid pressure $(\mathrm{Pa})$ and $\mathbf{g}$ the body accelerations acting on the continuum $\left(m s^{-2}\right) . \boldsymbol{F}_{\boldsymbol{E} \boldsymbol{H} \boldsymbol{D}}$ represents the electrical body force term of the EHD-effect $\left(\mathrm{N} \mathrm{m}^{-3}\right)$, which appears in form of electric wind in the flow field and is determined as follows:

$$
\boldsymbol{F}_{\boldsymbol{E H D}}=\boldsymbol{E} \rho_{s}
$$

The flow field is simulated with the flow simulation software OpenFOAM ${ }^{\circledR}$ based on the Finite Volume Method. A program interface between MATLAB $\AA$ and OpenFOAM ${ }^{\circledR}$ was developed to exchange input and output parameters in form of geometry and mesh data, boundary and start conditions, material and substance values as well as field data.

Geometry and mesh data are created in MATLAB ${ }^{\circledR}$ by the PDE Tool's automatic mesher and the finished mesh is transferred to the OpenFOAM ${ }^{\circledR}$ software. As a simplification, a two-dimensional geometry with a radial 
symmetry is assumed, see Fig. (4). The number of triangular cells of the mesh is also 4512.

For the implementation of the EHD effect the simpleFoam solver was modified. Flow simulations of stationary and incompressible Newtonian and turbulent fluids can be performed with the simpleFoam solver (OpenFOAM ${ }^{\circledR}$ User Guide), in which the standard k- $\epsilon$ model was used as turbulence model. The modified solver considers the influence of the electric wind as a body source term in the Navier-Stokes equation based on the current fields of $\boldsymbol{E}$ and $\rho_{s}$, also see Eq. (21) and Eq. (22). As $\boldsymbol{F}_{\boldsymbol{E} \boldsymbol{H} \boldsymbol{D}}$ is a spatial volume force in the flow field, it must be projected onto a two-dimensional geometry.

The resulting simulated flow field is then returned to the other MATLAB ${ }^{\circledR}$ models via the programmed interface.

The following boundary conditions are used for the flow and pressure field of the model:

\begin{tabular}{lll}
\hline Boundary & Description & Value \\
\hline B1 & inlet flow & $\boldsymbol{U}=\boldsymbol{U}_{\text {in }}$ \\
& & $\nabla p=0$ \\
\hline B3 & outlet flow & $\nabla \boldsymbol{U}=0$ \\
& & $p=p_{0}$ \\
\hline B2, B4, B5 & Wall & $\boldsymbol{U}=0$ (no slip) \\
& & $\nabla p=0$ \\
\hline
\end{tabular}

Table 3: Boundary conditions of the flow and pressure field.

In Tab. (3), $U_{\text {in }}$ corresponds to the inlet flow $\left(m s^{-1}\right)$ and $p_{0}$ to the ambient pressure $(\mathrm{Pa})$.

\subsection{Calculation sequence}

The calculation sequence shown in Fig. (6) starts with an input current $I_{0}$ and an input start voltage $V_{0}$ of the DE needle. This input voltage can be determined experimentally or estimated by using empirical formulas (e.g. according to Peek [8]). Next, the three model fields are calculated until convergence is achieved. After convergence, a correction value $\Delta \mathrm{V}$ is determined for the voltage potential of the DE needle via the resulting electrostatic field. Afterwards, the potential $V_{0}$ is adjusted accordingly with $V_{0}=V_{0}+\Delta V$ and the calculation of the fields is started again.

The calculation sequence ends as soon as the correction value $\Delta \mathrm{V}$ runs towards zero and no voltage potential change can be observed anymore.

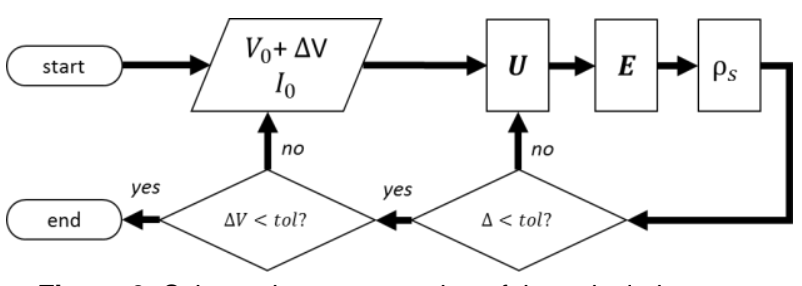

Figure 6: Schematic representation of the calculation sequence.

\section{Model validation}

The model is validated by comparing the experimentally determined and simulated voltage-current characteristics of the DE needle, which are shown in Fig. (7).

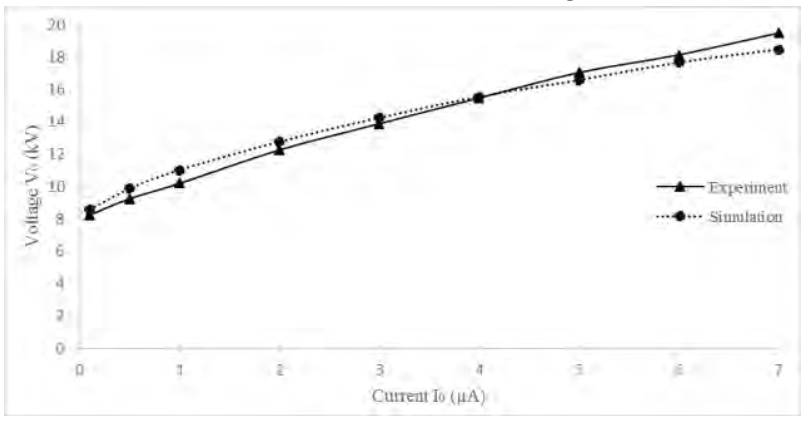

Figure 7: Voltage-current characteristics of the DE needle.

The breakdown voltage of the DE needle was approximately $V_{0}=8 \mathrm{kV}$ in the experiment. This value was used as the starting value for the simulation according to the calculation sequence. As can be seen in Fig. (7), the curve progression of the simulation largely complies well with the experimentally determined curve progression, whereby the simulation slightly exceeds the voltage potential below $4 \mu \mathrm{A}$ and slightly falls below it above $4 \mu \mathrm{A}$.

These deviations can probably be explained by inaccuracies in geometric modelling (e.g. the shape of the DE needle) and by the model simplifications of rotational symmetry that were applied.

\section{Conclusion and outlook}

Due to the various simplifications in geometry and symmetry assumptions, an efficient DE needle model could be developed, which provides fast and sufficiently good results with regard to validation.

Based on this, the particle flight can then be modelled using the Discrete Element Method (DEM) to analyse the particle separation behaviour by the DE needle in the electrostatic precipitator.

If the accuracy of the model is to be improved, a three-dimensional model approach to the space charge 
density as well as the flow field should be used. It would also be advisable to use a more precise geometric model. However, these improvements would be accompanied by an increased computing time.

Based on the model presented, the modelling of ozone production at the DE needle would also be an interesting topic for future studies.

\section{References}

[1] Caste GSP. Electrostatic Precipitation in Electrified Media and Positive Corona Ozone Generation in the Design of High Efficiency Air Cleaners [dissertation]. [Faculty of Engineering Science, CAN]. University of Western Ontario; 1969.

[2] Boelter KJ, Davidson JH. Ozone Generation by Indoor, Electrostatic Air Cleaners. Aerosol Sci. and Technology. 1997; 27(6), 689-708. doi: 10.1080/0278682970 8965505.

[3] Chen J, Davidson JH. Ozone production in the positive DC corona discharge: Model and comparison to experiments. Plasma Chem. and Plasma Proc. 2002; 22 (4), 495-522. doi: 10.1023/A:10231315412208

[4] Chen J, Davidson JH. Ozone production in the negative DC corona: the dependence of discharge polarity. Plasma Chem. and Plasma Proc. 2003; 23 (3), 501-518. doi: 10.1023/A:1022468803203

[5] Bo Z, Yu K, Lu G, Mao S, Chen J, Fan F-G. Nanoscale discharge electrode for minimizing ozone emission from indoor corona devices. Env. science \& technology. 2010; 44 (16), 6337-6342. doi: 10.1021 /es903917f.

[6] Hak-Joon K, Myungjoon K, Bangwoo H, Chang GW, Ayyoub Z, Noureddine Z, Yong-Jin K. Fine particle removal by a two-stage electrostatic precipitator with multiple ion-injection-type prechargers. J. of Aerosol Sciene. 2019; 130, 61-75. doi: 10.1016/j.jaerosci.2019.01.004.

[7] Leuchtmann P. Einführung in die elektrische Feldtheorie. 1. Auflage. Freising: Pearson Studium; 2007. 602 p.

[8] White HJ. Entstaubung industr. Gase mit Elektrofiltern. Leipzig: D. Verlag für Grundstoffindustrie; 1969. 336 p.

[9] McDonald JR, Smith WB, Spencer, Herbert W, Sparks, Leslie E. A mathematical model for calculating electrical conditions in wire-duct electrostatic precipitation devices. J. of Appl Phys. 1977; 48 (6), 2231-2243. doi: 10.1063/1.324034.

[10] Abdel-Salam M, Nakano M, Mizuno A. Corona-induced pressures, potentials, fields and currents in electrostatic precipitator configurations. J. Phys. D: Appl. Phys. 2007; 40 (7), 1919-1926. doi: 10.1088/0022-3727/40/7/014.

[11] Patankar SV, Minkowycz WJ, Sparrow EM. Series in computational methods in mechanics and thermal sciences. New York: McGraw-Hill Book Company; 1980, $197 \mathrm{p}$.

[12] Long Z, Yao Q, Song Q, Li S. A second-order accurate finite volume method for the computation of electrical conditions inside a wire-plate electrostatic precipitator on unstructured meshes. Journal of Electrostatics. 2009; 67 (4), 597-604. doi: 10.1016/j.elstat.2008.12.006.

[13] Chun YN, Chang J-S, Berezin AA, Mizeraczyk J. Numerical modeling of near corona wire electrohydrodynamic flow in a wire-plate electrostatic precipitator. IEEE Trans. Dielect. Electr. Insul. 2007; 14 (1), 119124. doi: 10.1109/TDEI.2007.302879.

[14] Long Z, Yao Q. Evaluation of various particle charging models for simulating particle dynamics in electrostatic precipitators. J. of Aer. Sci. 2010: 41 (7), 702-718. doi: 10.1016/j.jaerosci.2010.04.005.

[15] Chang JS, Dekowski J, Podlinski J, Brocilo D, Urashima K, Mizeraczyk J. Electrohydrodynamic gas flow regime map in a wire-plate electrostatic precipitator. Fourtieth IAS Ann..Meet. C. Record of the 2005 Ind. Appl. Conf. 2005; 4, 2597-2600. doi: 10.1109/IAS.2005.1518826.

[16] Launder BE, Spalding DB. The numerical computation of turbulent flows. C. Meth.in Appl. Me. and En. 1974; 3(2), 269-289. doi: 10.1016/0045-7825(74)90029-2. 